\title{
Autoestima y socialización maternal: Un análisis transcultural
}

Norma Ivonne González Arratia \& Marta Gil Lacruz

El autoconcepto y la autoestima resultan piezas claves en la explicación del funcionamiento y adaptación eficaz del individuo a su medio ambiente. El contexto de referencia, la cultura, la estratificación social, el sistema familiar, etcétera, a su vez, se constituyen en agentes causales de la socialización de este esquema. En este proceso, la figura materna sería especialmente importante en la transmisión de valores diferenciales y en la consolidación de una evaluación positiva de los hijos. Este artículo analiza desde una aproximación psicosocial el impacto de la influencia cultural en dicho pensamiento y afecto, estableciendo la comparación entre los rasgos con los que se describen las madres y sus respectivos hijos de dos muestras diferentes: España y México.

Autoconcepto / autoestima / identidad / cultura / socialización / valores

\section{Self-esteem and maternal socialization: a transcultural analysis}

Self-concept and self-esteem are two basic concepts that explain an individual's successful functioning and adaptation in her environment. Additionally, culture, social stratification, and family system constitute causal agents in the process of socialization. In this process of development, maternal figures become an important source of transmission of differential values and positive self-evaluation for their children. In this article, from a psycho-social perspective, the cultural influence of maternal figures is analyzed. A comparison between characteristics both mothers and children from samples of Spain and Mexico is made.

Self-concept / self-esteem / identity / culture / values

Correo electrónico: nigalf@mail.uaemex.mx/mglacruz@posta.unizar.es 


\section{INTRODUCCIÓN}

\section{Autoestima y autoconcepto}

La literatura científica sobre la definición del self suele distinguir entre el autoconcepto o la idea de sí mismo y la autoestima que se refiere a los sentimientos resultantes de dicha percepción (Wylie, 1961, 1979). Sin embargo, a menudo se utilizan indistintamente como términos complementarios dada su interrelación y causalidad recíproca (Pope, McHake \& Craighead, 1996).

De este modo, una elevada autoestima se fundamenta en una valoración positiva del propio autoconcepto y en el nivel de concordancia entre el autoconcepto percibido y el autoconcepto que se estima como ideal (Pope, McHake \& Craighead, 1996).

$\mathrm{El}$ autoconcepto se desarrolla en un determinado contexto social y cultural (Musitu, Roman \& Gracia, 1988). La importancia de factores psicosociales y culturales en la construcción de la identidad personal cuenta con un importante respaldo empírico (Rosenberg, 1979; Shrauger \& Schoeneman, 1979, Felson, 1981a, 1981b). Un ejemplo de esta corriente de investigación se dispone en el concepto de self social. Según Martínez y Montane (1981), este self abarcaría las percepciones que una persona tiene de los roles sociales que desempeña. Se trataría de la autoevaluación en función de unas cualidades subjetivables y valorativas. La persona se compara a sí misma y a los demás, en base a unos parámetros y cualidades que provienen de su experiencia social y son compartidas por los grupos de referencia como juicios de valor positivos o negativos (Soto, 1990).

El self como conjunto de categorías personales y sociales (Musitu, 1984) implica el compartir criterios de evaluación y normas de valor en función de los colectivos de adscripción. Por tanto, la estructura de valores puede servir para diferenciar culturas y los diferentes contenidos de la identidad que promueven (Triandis, 1985: 8895): la definición del uno mismo como independiente o dependiente del grupo, la prioridad de metas personales frente a metas grupales, el énfasis bien en las relaciones de intercambio o instrumentales, o bien en las interacciones comunales, el predominio de determinadas actitudes frente a las normas (ej. rebeldía, obediencia, etcétera), constituyen ejemplos de estas pautas compartidas. Incluso disponemos de referencias empíricas de cómo diferentes rasgos de personalidad y su correspondiente valoración cambian de una cultura a otra (Díaz-Loving, Díaz-Guerrero, Helmerich \& Spence, 1981; Shweder, Mahapatra \& Miller, 1990).

Las personas pertenecemos a grupos de los que obtenemos una identidad social resultado de nuestra experiencia compartida y consecuentemente, nuestra autoestima depende de la creación y el mantenimiento de las 
identidades sociales positivas y distintivas (Ros \& Gómez, 1997). Además una de las características más relevantes del proceso perceptivo del self de un individuo, estriba en la variedad de contextos o ámbitos en que dicha evaluación se lleva a cabo. De modo que si la valoración que uno hace de sí mismo es producto de la interacción con los otros, los diferentes contextos en que esta interación se produce estarán asociados a distintas evaluaciones sobre uno mismo (Herrero, Musitu, Gracia, 1995). Gutiérrez (1989) enfatiza en el aspecto cultural de este contenido. Por tanto, de entre las diferentes acepciones disponibles, podemos entender la autoestima como una disposición personal perdurable, caracterizada por la consistencia temporal -concepción del rasgo-y también como una variable resultante de la autoevaluación regulada por eventos ambientales -perspectiva situacional- (O’Malley \& Bachman, 1983; Jackson \& Paunomen, 1980).

Así, la autoestima de una persona es una estructura central para acercarnos a su propia concepción del mundo (Carver \& Scheier, 1981; Rogers, 1981). La autoestima contiene, por una parte, la imagen que la persona tiene de sí misma y de sus relaciones factuales con su ambiente, y, por otra, su jerarquía de valores y objetivos-meta, dichos valores forman un modelo coherente a través del cual la persona trata de planificar y organizar su propia vida dentro de los límites establecidos por el ambiente social (Roman, 1983). Dada esta complejidad, actualmente diversos autores (Delugach, Bracken, Bracken \& Schike, 1992; Romero, Luengo \& Otero, 1995) han subrayado que la autoestima es un constructo integrado por diferentes dimensiones (ej. autoestima social, académica) que han de ser tenidas en cuenta a la hora de explorar la interacción de esta autovaloración con otras variables.

Comprender dicha interacción (subrayando el componente afectivo de la autoestima) supone profundizar en el papel causal de los sentimientos en los pensamientos y conductas (Zajonc, 1980). La cognición y la emoción están interrelacionadas (Bunge, 1985). Así, las creencias acerca del ambiente y de uno mismo contienen aspectos tanto cognitivos como afectivos. Desde una aproximación cognitivo conductual (Craighead, Meyers \& Craighead, 1985) una pieza clave de esta interdependencia es el entorno (escuela, familia) donde se desarrollan las vivencias psicosociales.

De hecho, la autoestima de uno mismo también presenta consecuencias sociales. Por ejemplo, una autoestima saludable fundamenta una percepción vital y una competencia emocional que se refleja en la calidad de las relaciones. En cambio, una baja autoestima se encuentra presente en buena parte de los diagnósticos psiquiátricos y por tanto, en relaciones disfuncionales con el entorno (American Psychiatric Asso- 
ciation, 1987). La propia autoestima es un agente protector de situaciones estresantes: una elevada autoestima anima al sujeto a participar en un mundo de relaciones recíprocas, en el que se recibe y ofrece ayuda, posibilitándose el acceso al apoyo social disponible e incidiendo en la promoción del bienestar psicológico (Herrero, 1994).

\section{Socialización de la autoestima}

La construcción del autoconcepto y de la autoestima son procesos paralelos y complementarios que se producen a lo largo del ciclo vital de la persona. Los campos específicos relacionados con los sentimientos de autovaloración se inician durante la infancia mediante la interacción y dependencia del niño con su familia. Según Harter (1983), será en este primer periodo de la vida en el que se consoliden: relaciones adecuadas con los padres, el autocontrol del afecto negativo, la autoaceptación y la conducta social. La consistencia de las demandas en cada una de estas áreas será especialmente importante en los sentimientos de autoestima del adolescente cuando se esfuerza en sustentar su autoidentidad.

En la adolescencia, no solo la familia desempeña un papel causal, los amigos se constituyen en una fuente importante de feedback del propio self. Disponemos de evidencia empírica sobre las consecuencias positivas del nivel de contacto y proximidad experimentado con los amigos en esta etapa y la autoestima personal, el nivel de intimidad y la madurez social (Buhrmester, 1990). En los adolescentes una autoestima adecuada se relaciona positivamente con variables tan importantes para el desempeño eficaz de la persona en su entorno como: el nivel de confianza y comunicación, la existencia de un tono emocional positivo, un mayor control de los impulsos, el abordaje del mundo externo y menores niveles de problemas psicopatológicos (Claex, 1992).

Soto (1990) infiere cómo el desarrollo del self en la adolescencia y preadolescencia evidencia ya una estructura multidimensional, tras la revisión de trabajos sobre esta temática que contemplan análisis factoriales (Byrne \& Shavelson, 1986; Marsh, Byrne \& Shavelson, 1988). En la evolución de dicha estructura se encuentra como base la madurez socio-cognitiva: se aprende a generalizar y a realizar teorías sobre uno mismo a partir de la experiencia concreta y de las autoafirmaciones, en primera instancia ideas absolutas y específicas que evolucionan a cualidades interpersonales, simpatía, amabilidad, popularidad, valoradas socialmente en función de las circunstancias (Livesley \& Bromley, 1973).

La comprensión y la cognición social describen la capacidad de una persona para entender los puntos de vista de los demás. El ponerse en lugar del otro se desarrolla de forma espectacular durante el periodo escolar (especial- 
mente entre los 11 y los 13 años) e implica la comprensión de los pensamientos, sentimientos y formas de vivir del entorno físico, así como las características personales de cada individuo (Livesly \& Bromley, 1973; Shantz, 1983), a cambio el entorno le manifestará aprecio y aprobación y dicha consideración se reflejará en la propia estima (Asher, 1978). Tanto el autoconcepto como la autoestima supondrán una mezcla de elementos perceptivos y atribucionales en los que resulta difícil discernir dónde empieza el sujeto y dónde la influencia del contexto de referencia. En este sentido, la presente investigación intenta avanzar en el análisis del impacto cultural en la formación de la propia identidad y su estimación afectiva.

\section{Método}

En este artículo se seleccionó el autoconcepto concebido como la estructura mental de carácter psicosocial, conformada por tres dimensiones básicas: conductual, afectiva y física, tanto reales como ideales, que le permiten al sujeto interactuar con el medio ambiente interno y externo que le rodea y que se encuentra mediatizado por la presencia de factores culturales. La autoestima se expresaría en la distancia observada entre el propio autoconcepto percibido y el autoconcepto que se pretende como ideal (Valdez \& Reyes, 1992; Valdez, González, Reyes \& Gil, 1996).

\section{Muestra}

A través de un muestreo no probabilístico de cuotas, se seleccionaron 363 sujetos residentes en las ciudades de Zaragoza (España) y Toluca (México). La muestra mexicana consta de 83 niños (40 varones, 43 mujeres) en edades comprendidas entre los 10 y los 13 años que cursan sexto año de primaria y sus respectivas madres. En la muestra española se evaluó a 100 madres y sus respectivos niños (56 varones, $44 \mathrm{mu}-$ jeres) de las mismas edades y en el mismo nivel escolar. En ambos casos, el cuestionario fue impartido en una situación de aula y se contó con la colaboración del equipo docente.

\section{Instrumento}

El instrumento utilizado fue el Autoconcepto de Valdez (1994), que consta de 37 reactivos (adjetivos) con cinco opciones de respuesta tipo Likert, en las que el sujeto debe posicionarse desde un continuo de totalmente de acuerdo a nada, en función de dos cuestiones: primero, como identifican dichos adjetivos su propio autoconcepto y segundo, hasta qué punto les gustaría tener dicha característica en su propia personalidad.

Este cuestionario fue seleccionado en base a su coherencia teórica planteada en la introducción, a sus características psicométricas $(\mathrm{a}=0,82$ Alpha de Cronbach) y a su relevancia cultural (Valdez \& Reyes, 1992). No obstante, antes de su contestación por parte de la 
muestra española fue necesario adaptar el vocabulario de cinco ítems: enojónenojadizo, travieso-latoso, platicadorhablador, necio-terco y acomedido-servicial).

\section{Procedimiento}

Tras la aplicación del instrumento y con el fin de realizar la pertinente factorialización (mediante un análisis factorial de componentes principales con rotación varimax) se agruparon los ítems en seis dimensiones que compartieron ambas muestras: S.N.: Social Normativo: S.E.: Social Expresivo; E.A.: Expresivo Afectivo, E.M.: Ético Moral, T.I.: Trabajo Intelectual, R.: Rebeldía.

A partir de esta dimensionalización, se procedió a realizar un análisis de varianza de una sola vía, para observar las diferencias entre medias para cada uno de los ítems. De esta forma, observamos que los sujetos mexicanos tienden a percibirse como más desobedientes, enojadizos, estrictos, estudiosos, tercos, traviesos y volubles en comparación con los niños españoles que se describieron en los siguientes términos: amables, bromistas y simpáticos.
Con relación a las madres, se encontraron diferencias significativas entre los adjetivos con los que se definían. Las madres mexicanas se identificaban con los siguientes reactivos: enojadizas, mentirosas, agresivas, estrictas y criticonas; las mujeres españolas: sinceras, simpáticas, tercas, aplicadas, amigables, románticas y buenas.

\section{Resultados}

A través de los análisis de varianza de una sola vía, se encontraron diferencias estadísticamente significativas $(\mathrm{p}>=$ $0,5)$ entre madres e hijos mexicanos, y como puede observarse en la tabla 1, las madres tienden a percibirse como amables, honestas, enojadizas, activas, limpias, respetuosas, mientras que sus hijos se ven a sí mismos en otros términos: traviesos, mentirosos, volubles, tercos.

En la muestra española también se utilizó este procedimiento con el objetivo de hacer la comparación generacional, destacando en el caso de las madres un rasgo diferencial con respecto a sus hijos, la sinceridad. A su vez, los niños españoles se consideraron en parámetros de desobediencia, rebeldía, agresividad, etcétera. 
Tabla 1

Análisis de varianza comparaciones madre-hijo autoconcepto real muestra mexicana

\begin{tabular}{lccccc}
\hline \multicolumn{1}{c}{ Variable } & $\mathrm{P}$ & $\overline{\mathrm{X}}$ Hijo & $\mathrm{DS}$ & $\overline{\mathrm{X}}$ Madre & $\mathrm{DS}$ \\
\hline Amable & .000 & 2.92 & .78 & $3.37^{*}$ & .63 \\
Honesto & .000 & 2.69 & .86 & $3.63^{*}$ & .63 \\
Relajiento & .048 & $2.60^{*}$ & 1.3 & 2.20 & 1.32 \\
Enojón & .044 & 2.34 & 1.1 & $2.66^{*}$ & 1.06 \\
Activo & .000 & 3.03 & 1.0 & $3.47^{*}$ & .73 \\
Limpio & .048 & 3.40 & .94 & $3.66^{*}$ & .74 \\
Sincero & .000 & $2.94^{*}$ & 1.1 & $3.51^{*}$ & .77 \\
Travieso & .000 & $2.90^{*}$ & 1.2 & 2.50 & 1.41 \\
Mentiroso & .001 & $2.02^{*}$ & .86. & 1.35 & 1.18 \\
Estudioso & .003 & $2.84^{*}$ & .98 & 2.37 & 1.26 \\
Leal & .000 & 3.11 & .81 & $3.66^{*}$ & .68 \\
Respetuoso & .000 & 3.32 & 1.2 & $3.78^{*}$ & .50 \\
Voluble & .000 & $2.44^{*}$ & 1.2 & 1.44 & 1.43 \\
Necio & .044 & 2.10 & 1.2 & $3.72^{*}$ & 1.41 \\
Romántico & .000 & 2.22 & 1.4 & 1.28 & 1.19 \\
Desobediente & .000 & $2.97^{*}$ & 1.2 & 1.62 & 1.27 \\
Ordenado & .029 & 3.09 & .97 & $3.43^{*}$ & .85 \\
Atento & .002 & 2.92 & 1.1 & $3.40^{*}$ & .82 \\
Acomedido & .001 & 2.91 & 1.1 & $3.39^{*}$ & .84 \\
Sentimental & .001 & 2.80 & 1.3 & $3.16^{*}$ & .92 \\
Estricto & .000 & 2.17 & 1.3 & $3.63^{*}$ & .96 \\
Trabajador & .000 & 2.15 & 1.0 & $2.85^{*}$ & 74 \\
Bromista & .017 & $1.36^{*}$ & 1.3 & 1.26 & 1.37 \\
Responsable & .000 & 2.01 & 1.1 & $3.28^{*}$ & .53 \\
\hline R & & & & & \\
\hline
\end{tabular}

$\mathrm{n}=183 \mathrm{gl}=182$

Tabla 2

Análisis de varianza comparaciones madre-hijo autoconcepto real muestra española

\begin{tabular}{lcccccc}
\hline Variable & $\mathrm{P}$ & $\mathrm{F}$ & $\overline{\mathrm{X}}$ Hijo & DS & $\overline{\mathrm{X}}$ Madre & $\mathrm{DS}$ \\
\hline Relajiento & .0348 & 4.542 & $3.36^{*}$ & 1.42 & 2.50 & 1.55 \\
Sincero & .0165 & 5.885 & 1.88 & 1.07 & $2.64^{*}$ & 1.44 \\
Mentiroso & .0013 & 10.80 & $3.13^{*}$ & 1.40 & 1.85 & 1.09 \\
Desobediente & .0201 & 5.528 & $3.10^{*}$ & 1.31 & 2.21 & 1.57 \\
Agresivo & .0005 & 12.56 & $3.33^{*}$ & 1.50 & 1.85 & 1.23 \\
Rebelde & .0528 & 3.814 & $3.70^{*}$ & 4.20 & 1.50 & 1.09 \\
Estricto & .0007 & 12.04 & $2.97^{*}$ & 1.31 & 1.71 & 1.06 \\
Criticón & .0155 & 6.000 & $3.24^{*}$ & 1.38 & 2.28 & 1.43 \\
\hline
\end{tabular}

$\mathrm{n}=\mathbf{2 0 0} \mathrm{gl}=199$ 
Tabla 3

Análisis de varianza comparaciones madre hijo autoconcepto ideal

muestra mexicana

\begin{tabular}{lcclccc}
\hline Variable & $\mathrm{P}$ & $\mathrm{F}$ & $\overline{\mathrm{X}}$ Hijo & $\mathrm{DS}$ & $\overline{\mathrm{X}}$ Madre & $\mathrm{DS}$ \\
\hline Platicador & .000 & 6.01 & 1.45 & 1.5 & $2.72^{*}$ & 1.3 \\
Enojón & .007 & 10.3 & 0.86 & 1.3 & $1.41^{*}$ & 1.5 \\
Respetuoso & .039 & 8.19 & 3.67 & .75 & $3.85^{*}$ & .55 \\
Voluble & .000 & 3.35 & $2.15^{*}$ & 1.5 & 1.09 & 1.4 \\
Aplicado & .014 & 4.68 & $3.64^{*}$ & .82 & 3.40 & 1.0 \\
Romántico & .008 & 7.62 & 2.76 & 1.4 & $3.31^{*}$ & 1.1 \\
Acomedido & .001 & 4.7 & 3.27 & 1.2 & $3.74^{*}$ & .56 \\
Cariñoso & .033 & 3.9 & 3.51 & .99 & $3.78^{*}$ & .54 \\
Estricto & .000 & 3.5 & 2.37 & 1.5 & $3.33^{*}$ & .89 \\
Inteligente & .046 & 3.8 & $3.63^{*}$ & .83 & 3.43 & 1.0 \\
\hline
\end{tabular}

$\mathrm{n}=183 \quad \mathrm{gl}=182$

De acuerdo con la autoestima entre madres e hijos, se observó que las madres mexicanas, poseen una autoestima más favorable que sus hijos, ya que, al aplicar la prueba t de Student, las medias indican que en el caso del grupo de las madres, existe menor distancia entre el autoconcepto real y el autoconcepto ideal, ya que se obtuvo para el grupo de madres una $\bar{X}=30.06$ y en el caso de sus hijos, en donde hay mayor distancia entre lo que son y lo que les gustaría ser con una $\bar{X}=27.31$ con un nivel de significatividad de $\mathrm{p}>=.05$.

Por el contrario, en relación con la autoestima entre madres e hijos españoles, se observó que los niños españoles, poseen una autoestima ligeramente más favorable que sus respectivas madres, ya que al aplicar la prueba $t$ de

Tabla 4

Análisis de varianza comparaciones madre hijo autoconcepto ideal muestra española

\begin{tabular}{lcccccc}
\hline Variable & $\mathrm{P}$ & $\mathrm{F}$ & $\overline{\mathrm{X}}$ Hijo & $\mathrm{DS}$ & $\overline{\mathrm{X}}$ Madre & $\mathrm{DS}$ \\
\hline Amable & .0001 & 15.36 & $3.56^{*}$ & .98 & 2.36 & 1.73 \\
Enojón/enojadizo & .0000 & 23.83 & 1.23 & 1.53 & $3.29^{*}$ & 1.06 \\
Activo & .0380 & 4.38 & $3.81^{*}$ & 3.57 & 0.29 & 10.82 \\
Limpio & .0002 & 14.86 & 1.76 & .604 & $3.00^{*}$ & 1.51 \\
Travieso & .0091 & 6.99 & $3.59^{*}$ & 1.59 & 2.93 & 1.38 \\
Simpático & .0000 & 19.39 & 1.33 & 1.02 & $2.22^{*}$ & 1.67 \\
Necio & .0001 & 15.63 & 1.11 & 1.60 & $3.08^{*}$ & 1.20 \\
Desobediente & .0015 & 10.52 & $3.71^{*}$ & 1.52 & 2.50 & 1.60 \\
Ordenado & .0211 & 5.44 & 1.38 & .87 & $3.08^{*}$ & 1.54 \\
Rebelde & .0005 & 12.89 & $3.29^{*}$ & 1.53 & 2.93 & 1.54 \\
Atento & .0136 & 6.25 & $3.67^{*}$ & 3.30 & 0.43 & 8.35 \\
Responsable & .0139 & 6.20 & $3.67^{*}$ & .826 & 3.08 & 1.07 \\
\hline $\mathrm{n}=200 \quad \mathrm{gl=199}$ & & & & & &
\end{tabular}


Student se encontró que los niños obtuvieron una $\overline{\mathrm{X}}=32.11$, mientras que las madres una $\bar{X}=29.19(p>=.05)$. Estos resultados indican que en el caso del grupo de los niños existe menor distancia entre el autoconcepto real y el autoconcepto ideal que en el caso de sus madres, en donde hay mayor distancia entre lo que son y lo que les gustaría ser.

\section{DISCUSIÓN}

Tal y como se expuso en el marco teórico, respecto a los datos obtenidos entre los niños y sus madres mexicanas y españolas, se constató que la estructura y el contenido del autoconcepto varían en función de la cultura de adscripción (Díaz-Loving, Díaz-Guerrero, Helmereich \& Spence, 1981). En la cultura mexicana, y de manera coherente con los resultados obtenidos, Díaz-Guerrero (1989) plantea la importancia de valores y normas sociales que conllevan habilidades sociales de relación, convivencia armoniosa e incluso la prioridad de los aspectos afectivos en las relaciones. En cambio, en los sujetos españoles, en este caso niños, el ser social normativo supone primar el orden y la productividad en un marco de respeto en la interacción.

Estos datos se reflejan claramente en una serie de factores, como la rebeldía, que en los niños españoles supone la primera nota definitoria y la tercera en México. En los niños españoles este comportamiento se relaciona con la autoafirmación, la agresividad, dominancia y asertividad, frente a los niños mexicanos que lo asocian con un comportamiento disruptivo y por tanto susceptible de rechazo en el entorno (Flores, Díaz, Guzmán, Bárcenas \& Godoy, 1992). En las dimensiones de trabajo intelectual y expresión afectiva encontramos tendencias similares, ya que el propio concepto de trabajo se define culturalmente en México como obediencia y afiliación, y en España los niños lo relacionan con ser aplicado y estar atento.

En relación con la figura materna, aunque ambos países comparten como nota distintiva la importancia de ser cariñosa, buena, amigable y simpática (dimensión social expresiva), en las mujeres españolas observamos un cambio de tendencia hacia una definición instrumental del propio autoconcepto: trabajadora, activa, aplicada. En las mujeres mexicanas se constató la presencia de un mayor número de rasgos negativos para evaluar su propia identidad: agresivas, mentirosas, criticonas. Díaz-Guerrero (1989) interpreta este fenómeno dentro de una posible tendencia cultural del mexicano a infravalorarse.

Sin embargo, en relación con la autoestima, las tendencias parecen invertirse en función del país de procedencia. Las madres mexicanas presentan una menor distancia entre su autoconcepto real e ideal en comparación 
con sus hijos, en los que se da una menor autoestima. Autores como Dolto (1990) explican este hecho en función de la edad de desarrollo físico e intelectual del niño, la adolescencia es una etapa de crisis y de cuestionamiento en el que la propia autoestima puede resentirse. Por el contrario, las madres de la muestra española se valoran menos que sus propios hijos.

Según González-Arratia (1998), estas diferencias de autoestima pueden tener dos explicaciones: la primera es que los niños se perciben con características que denotan claramente su rebeldía y posiblemente su proceso madurativo todavía no ha hecho impacto en su propia identidad. Una hipótesis alternativa es que las madres españolas utilizan más características expresivas, socialmente aceptadas en relación con el estereotipo femenino.

Por tanto, este trabajo respalda la evidencia empírica que informa de una relación significativa respecto a las estructuras de autoconcepto y autoestima entre madres e hijos. Especialmente en los sujetos mexicanos, donde los resultados apuntan a una convergencia entre el autoconcepto y autoestima en ambos elementos de la díada. La distancia entre autoconcepto real e ideal mexicano es mínima, a los niños y las madres les gusta ser tal como son y sus ideales no son tan elevados como en los niños y madres españoles.

Además, tanto las madres como sus hijos (independientemente de la cultura de adscripción) realizan su propia evaluación en función del papel que desempeñan, lo que implica que tanto el autoconcepto como la autoestima pueden ser modificables a través del tiempo y de acuerdo con las circunstancias. No obstante, a pesar de que este estudio evidencia el impacto cultural en la formación del autoconcepto, resulta indispensable realizar otras investigaciones para ampliar y complementar estos resultados. Variables como el nivel socioeconómico, tipo de escuela (pública, privada, urbana, rural), rendimiento escolar, autoconcepto de ambos padres, ambiente familiar, edad, otros países, etcétera, aportarían nuevas luces a esta temática.

\section{REFERENCIAS}

American Psychiatric Association (1987). Diagnostic and statistical manual of mental disorders. Washington, D.C.: American Psychiatric Association.

Asher, S. (1978). Children for relation. En: M. Lamb (Ed.). Sociopersonality development. Nueva York: Holt.

Bunge, M. (1985). El problema mente-cerebro: Un enfoque psicobiológico. Barcelona: Tecnos.

Buhrmester, D. (1990). Intimacy of friendship, interpersonal competence, and adjustment during preadolescence and adolescence. Child development, 61, 1101-1111.

Byrne, B. \& Shavelson, R. (1986). On the structure of adolescent self-concept. Journal of Educational Psychology, 78, 474-481. 
Carver, C. S. \& Scheier, M. F. (1981). Attention and self-regulation: a control theory approach to human behavior. Nueva York: Springer.

Claex, M. (1992). Friendship and personal adjustement in adolescence. Journal of Adolescent, 15, 39-55.

Craighead, W. E.; Meyer, A. W. \& Craighead, L. W. (1985). A conceptual model for cognitive-behavior therapy with children. Journal of Abnormal Child Psychology, 13 (3), 331-342.

Delugach, R. R.; Bracken, B. A.; Bracken, M. J. \& Schike, M. C. (1992). Self concept: multidimensional construct exploration. Psychology in the School, 29, 213-223.

Díaz-Guerrero, R. (1989). Psicología del mexicano. México: Trillas.

Díaz-Loving, R.; Díaz-Guerrero, R.; Helmereich, R. \& Spence, J. (1981). Comparación transcultural y análisis psicométrico de una medida de rasgos masculinos y femeninos. Revista de la Asociación Latinoamericana de Psicología Social, 1, 3-38.

Dolto, F. (1990). La causa de los adolescentes. México: Seix-Barral.

Felson, R. B. (1981a). Ambiguity and bias in the self concept. Social Psychology Quarterly, 44(1), 64-69.

Felson, R. B. (1981b). Self and reflected appraisals among football players: a test of the median hypothesis. Social Psychology Quarterly, 44, 116-126.

Flores, G. M.: Díaz, L. R.; Guzmán, P. L.; Bárcenas, M. G. \& Godoy, P. G. (1992). Asertividad, abnegación y agresividad: Evaluación semántica. En: La psicología social en México, vol. IV, 303-308.
González-Arratia, N. I. (1998). Autoconcepto y autoestima en madres e hijos de sexto año de primaria, en sujetos mexicanos y españoles. Informe académico. Toluca: Universidad Autónoma del Estado de México, Facultad de Ciencias de la Conducta.

Gutiérrez, M. (1989). Interacción familiar: autoconcepto y conducta prosocial. En: C. Soto (1990). Autoconcepto y autoeficacia en adolescentes. Tesis de licenciatura. Universidad de Zaragoza, Facultad de Psicología.

Harter, S. (1983). Development perspectives on the self system. En: A. W. Pope; S. M. McHale \& W. E. Craighead. (1996). Mejora de la autoestima: Técnicas para niños y adolescentes. Barcelona: Martínez Roca.

Herrero, J. (1994). Estresores sociales y recursos sociales: el papel del apoyo social en el ajuste bio-psico-social. Tesis doctoral. Valencia: Universitat de Valencia.

Herrero, J.; Musitu, G. \& Gracia, E. (1995). Autoestima y depresión: Relaciones directas versus indirectas. Revista de Psicología Social, 10(2), 191-204.

Jackson, D. N. \& Paunomen, S. V. (1980). Personality structure and assessment. En: C. Soto (1990). Autoconcepto y autoeficacia en adolescentes. Tesis de licenciatura. Universidad de Zaragoza, Facultad de Psicología.

Livesly, W. \& Bromley, D. (1973). Person perception in childhood and adolescence. Londres: Wiley.

Marsh, H.; Byrne, B. \& Shavelson, R. (1988). A multifaceted academic selfconcept: its hierarchical structure and its relation to academic achievement. 
Journal of Educational Psychology, 81, 57-69.

Marsh, H. \& Hocevar, D. (1985). The application of confirmatory factor analysis to the study of self concept: first and higher order factor structures and their invariance across age groups. Psychology Bulletin, 97, 562-582.

Martínez, C. \& Montane, J. (1981). Nivel de autoestima, relaciones intrafamiliares y distorsión de la percepción en un contexto ambivalente. Actas del Congreso de la Sociedad Valenciana de Análisis y Cambio de Conducta. Alicante.

Musitu, G. (1984). Interacción familiar y autoestima según el sexo: Bases para una intervención. I Simposium Nacional de Terapia Familiar. Madrid.

Musitu, G.; Roman, J. M. \& Gracia, E. (1988). Familia y educación. Barcelona: Labor.

O’Malley, P. \& Bachman, J. (1983). Selfesteem: change and stability between ages band 23. Developmental Psychology, 19 (2), 257-268.

Pope, A. W.; McHale, S. M. \& Craighead, W. E. (1996). Mejora de la autoestima: Técnicas para niños y adolescentes. Barcelona: Martínez Roca.

Rogers, T. B. (1981). A model of the self as an aspect of the human information processing system. En: G. Musitu; J. M. Roman \& E. Gracia (1988). Familia y educación. Barcelona: Labor.

Roman, J. M. (Ed.) (1983). Análisis transaccional: Modelo y aplicaciones. Barcelona: CEAC.

Romero, E.; Luengo, M. A. \& Otero, J. M. (1995). La relación entre autoestima y consumo de drogas en los adolescentes: Un análisis longitudinal. Revista de Psicología Social, 10(2), 149-160.

Ros, M. \& Gómez, A. (1997). Valores personales individualistas y colectivistas y su relación con la autestima grupal. $R e$ vista de Psicología Social, 12(2), 129-256.

Rosenberg, M. (1979). Conceiving the self. Nueva York: Basic Books.

Shweder, R. A.; Mahapatra, M. \& Miller, J. G. (1990). Culture an Moral Development. En: J. W. Stiegler; R. A. Shweder \& G. Herdt (Eds). Culture Psychology. Nueva York: Cambridge University Press.

Shantz, C. (1983). Social cognition. En: A. W. Pope; S. M. McHale \& W. E. Craighead (1996). Mejora de la autoestima: Técnicas para niños y adolescentes. Barcelona: Martínez Roca.

Shrauger, J. S. \& Schoeneman, T. J. (1979). The symbolic interactionist' view of self concept: though the looking-glass darkly. Psychology Bulletin, 86, 549-573.

Soto, C. (1990). Autoconcepto y autoeficacia en adolescentes. Tesis de licenciatura. Universidad de Zaragoza, Facultad de Psicología.

Triandis, H. C.; Leung, K.; Villareal, M. J. \& Clarck, F. L. (1985). Allocentric versus idiocentric tendencies: convergent and discriminat validation. Journal of Research in Personality, 19, 395-415.

Triandis, H. C.; Bontempo, R. \& Villareal, M. J. (1988). Individualism and collectivism: cross cultural perspectives on self-ingroup relationships. Journal of Personality and Social Psychology, 54 (2), 323-338. 
Triandis, H. C. (1995). Horizontal and vertical individualism and collectivism and work. Work Paper, 94(11), 047/6. The Netherlands: Tilburg University.

Valdez, J. L. \& Reyes, Y. (1992). Las redes semánticas y el autoconcepto. La Psicología Social en México, vol. IV, 193-199.

Valdez, J. L.; González, Y.; Reyes, Y. \& Gil, M. (1996). El autoconcepto en ni- ños mexicanos y españoles. Revista Interamericana de Psicología, 2(30), 179-188.

Wylie, R. C. (1961). The self concept. University of Nebraska Press. Lincon and London.

Wylie, R. C. (1979). The self concept (vol. 2). Theory and research on selected topics. University of Nebraska Press. Lincon and London. 